\title{
Performance Evaluation of Distributed Mobility Management Protocols: Limitations and Solutions for Future Mobile Networks
}

\author{
J. Carmona-Murillo, ${ }^{1}$ I. Soto, ${ }^{2}$ F. J. Rodríguez-Pérez, ${ }^{1}$ \\ D. Cortés-Polo, ${ }^{3}$ and J. L. González-Sánchez ${ }^{3}$ \\ ${ }^{1}$ Departamento de Ingeniería de Sistemas Informáticos y Telemáticos, Universidad de Extremadura, Extremadura, Spain \\ ${ }^{2}$ Departamento de Ingeniería Telemática, Universidad Carlos III de Madrid, Madrid, Spain \\ ${ }^{3}$ Centro Extremeño de Investigación, Innovación Tecnológica y Supercomputación, Cáceres, Spain
}

Correspondence should be addressed to J. Carmona-Murillo; jcarmur@unex.es

Received 5 October 2016; Accepted 18 January 2017; Published 16 February 2017

Academic Editor: Juan C. Cano

Copyright (C) 2017 J. Carmona-Murillo et al. This is an open access article distributed under the Creative Commons Attribution License, which permits unrestricted use, distribution, and reproduction in any medium, provided the original work is properly cited.

\begin{abstract}
Mobile Internet data traffic has experienced an exponential growth over the last few years due to the rise of demanding multimedia content and the increasing number of mobile devices. Seamless mobility support at the IP level is envisioned as a key architectural requirement in order to deal with the ever-increasing demand for data and to efficiently utilize a plethora of different wireless access networks. Current efforts from both industry and academia aim to evolve the mobility management protocols towards a more distributed operation to tackle shortcomings of fully centralized approaches. However, distributed solutions face several challenges that can result in lower performance which might affect real-time and multimedia applications. In this paper, we conduct an analytical and simulated evaluation of the main centralized and proposed Distributed Mobility Management (DMM) solutions. Our results show that, in some scenarios, when users move at high speed and/or when the mobile node is running long-lasting applications, the DMM approaches incur high signaling cost and long handover latency.
\end{abstract}

\section{Introduction}

Over the last few years, the enormous proliferation of powerful mobile devices has created a high demand for mobile data traffic that grows year by year. In fact, recent reports outline the notion that this traffic will grow nearly eightfold between 2015 and 2020 [1]. Moreover, as mobile data traffic increases, the growth in signaling load is expected to increase almost $50 \%$ faster than the growth in data traffic over the next few years [2]. As a result, during these years, operators, industry, and the research community have been evolving their mobile solutions to tackle such challenges, especially at the standardization organizations.

To provide continuous service to mobile users, even if they change their point of attachment to the network, numerous IP mobility management protocols have been standardized. The Internet Engineering Task Force (IETF) proposed centralized mobility support solutions for all-IP networks, where centralized mobility anchors manage the traffic and signaling of the mobile nodes (MNs). The most representative centralized mobility management $(\mathrm{CMM})$ protocols are Mobile IPv6 (MIPv6) [3] and Proxy Mobile IPv6 (PMIPv6) [4]. MIPv6 introduces a home agent (HA) that acts as the mobility anchor (MA), while PMIPv6 manages mobility locally by introducing a Local Mobility Anchor (LMA) in the domain. In CMM, this mobility anchor is responsible for both mobility signaling and user data forwarding.

Centralized approaches are likely to have several shortcomings or limitations, which require costly network dimensioning and engineering to resolve. The main problems that have been previously identified concerning centralized solutions are nonoptimal routing, scalability issues, and excessive signaling overhead, which implies longer handover latencies and vulnerabilities due to the existence of a single 
point of failure [5]. With these limitations, the IETF has recently proposed the Distributed Mobility Management (DMM) paradigm, which can be deemed as a novel and promising approach to handle mobility [6]. In DMM, the core idea is that the mobility anchors are distributed within the network, topologically closer to the users, with the aim of providing almost optimal routing support and an efficient use of network resources to improve the scalability required for next generation mobile networks [7].

However, despite the fact that a number of mobility management approaches are being designed towards a more distributed operation aiming to mitigate problems related to centralized operation, there are instances where DMM incurs higher costs and the performance of the network might be compromised.

In this article, we develop an analytical framework that allows the evaluation and comparison of mobility protocols in order to identify the benefits and limitations of the DMM paradigm. This analytical model is an extension of our previous work [8], adding many refinements and extensions to achieve a unified model which can evaluate both centralized and distributed mobility protocols in terms of mobility costs and handover performance. Additionally, the proposed model uses a new parameter, namely, Mobility Anchor Load (MAL), which is defined as the current data generated by a mobile node (MN) to the number of mobility anchors associated with the MN, to observe the average load of each of the different anchors distributed across the network. We consider MIPv6 and PMIPv6 as two main representatives for the centralized mobility management paradigm and Host-Based DMM [9], Network-Based DMM [10], and our DM3 (Distributed Mobility Management MPLS) proposal [8] as the distributed schemes. In addition to the analytical evaluation, the model is validated through simulation. Our numerical results demonstrate that there are instances where DMM incurs higher signaling costs and longer handover latency than CMM protocols, which can compromise the performance of the network. These situations are those in which users are moving at a high speed and the MN is running long-lasting applications. Even under these conditions, our DMM solution mitigates the limitations of DMM approaches and generally outperforms both the centralized and the distributed schemes.

The rest of the paper is organized as follows. In Section 2, we briefly present background information about centralized and Distributed Mobility Management protocols. Then, Section 3 describes the analytical model used to compare CMM and DMM solutions. The results of the numerical analysis are given in Section 4 and Section 5 presents the performance evaluation through simulation. Finally, Section 6 concludes the paper.

\section{Background}

2.1. Centralized Mobility Management. In order to enable seamless mobility in MIPv6 [11] (see Figure 1), the mobile node $(\mathrm{MN})$ is assigned with a permanent home address in its Home Network (HN) and establishes a connection with the communication peer, the Correspondent Node (CN). A Home Agent (HA) serves as the anchor node in the HN that tracks the network connection point (location) of a user as the user moves. Periodically, or whenever the MN changes its point of attachment to the network, the $\mathrm{MN}$ registers a new care-of-address (CoA) with the HA through Binding Update (BU) messages, informing of its current location and establishing a tunnel between the $\mathrm{HA}$ and the MN located in a visited network. In MIPv6, the HA is the centralized part of the system since it is on the critical path of both signaling and data forwarding for mobile users.

For its part, PMIPv6 has been standardized as an extension to MIPv6. PMIPv6 introduces two network entities, namely, the Local Mobility Anchor (LMA) and the Mobile Access Gateway (MAG). The former is the home agent for the MN in a PMIPv6 domain, where the mobility is managed by PMIPv6. The latter is an access router (AR) that manages the mobility-related signaling for an $\mathrm{MN}$ that is attached to it. When a handover occurs from one MAG to another, the binding is updated at the LMA by means of Proxy Binding Update (PBU) and Proxy Binding Acknowledgment (PBA) messages. In this procedure, the LMA updates the associated Binding Cache Entry, which contains, among other information, the mobile node identifier, the Home Network prefix, and the Proxy Care-of-Address (PCoA), which is the MAG's address according to where the $\mathrm{MN}$ is currently attached. Once the MN is registered, the LMA is its topological anchor point for the IPv6 prefix that is uniquely assigned to the MN (Home Network Prefix). As a result of the registration, an IP bidirectional tunnel is created between the LMA and the MAG, so mobility is provided in a transparent way to the user. This operation of PMIPv6 is shown in Figure 2.

2.2. Distributed Mobility Management. As briefly alluded to above, CMM requires a single handed mobility anchor, for example, HA at MIPv6 and LMA at PMIPv6, to allow for session continuity when MNs are moving across different networks. Nowadays, most of the deployed architectures, such as 3GPP networks, have a small number of centralized anchors managing the traffic of thousands of mobile users.

These centralized approaches have certain limitations for handling a large volume of mobile data traffic such as nonoptimal routing, scalability problems, and reliability/robustness [5]. In order to address these limitations, a new paradigm has been recently proposed which has gained attention: the socalled Distributed Mobility Management. In essence, DMM develops a new concept for handling mobility, with the main characteristics being that the anchors are placed closer (topologically) to the user, distributing the control and data plane mobility functions among entities located at different places in the core/access network.

A representative proposal of a DMM solution which is based on Mobile IPv6 is detailed in [9] (Host-Based DMM, HB-DMM). In this work, the authors attempt to improve the performance of mobility support by extending the MIPv6's HA to the AMA (Access Mobility Anchor), which is a new mobility anchor defined for the proposed Host-Based DMM approach. These AMAs are distributed at 


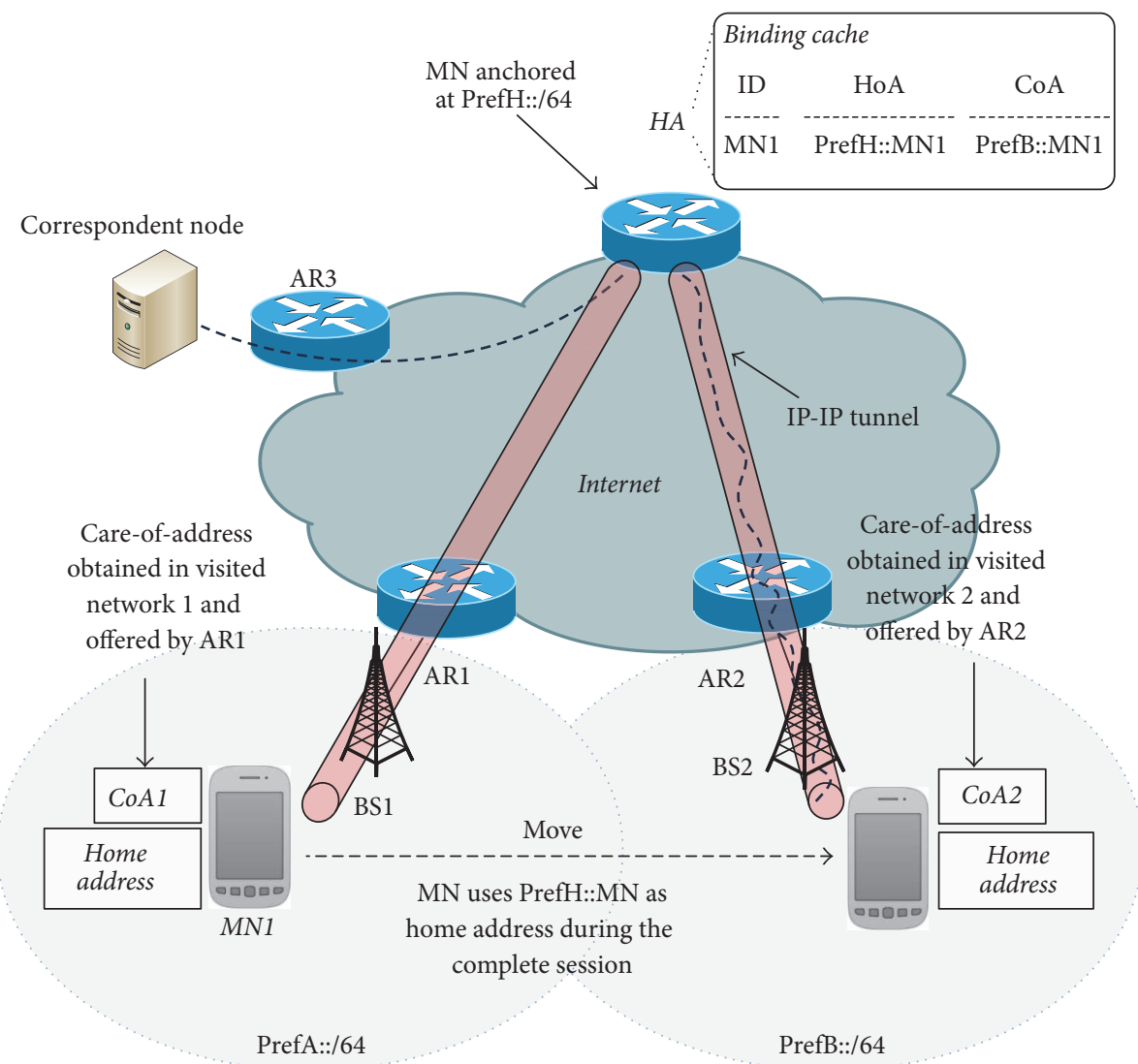

Figure 1: Mobile IPv6 operation.

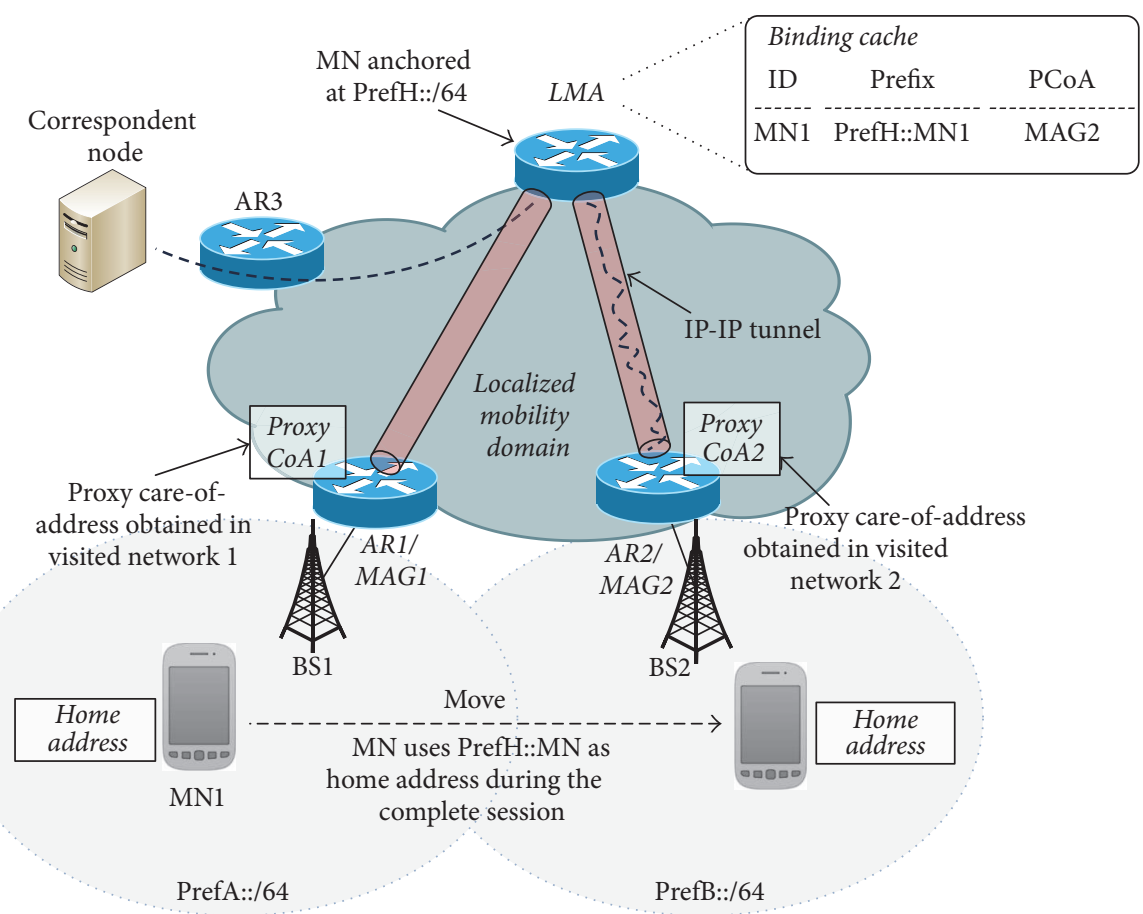

Figure 2: Proxy Mobile IPv6 operation. 


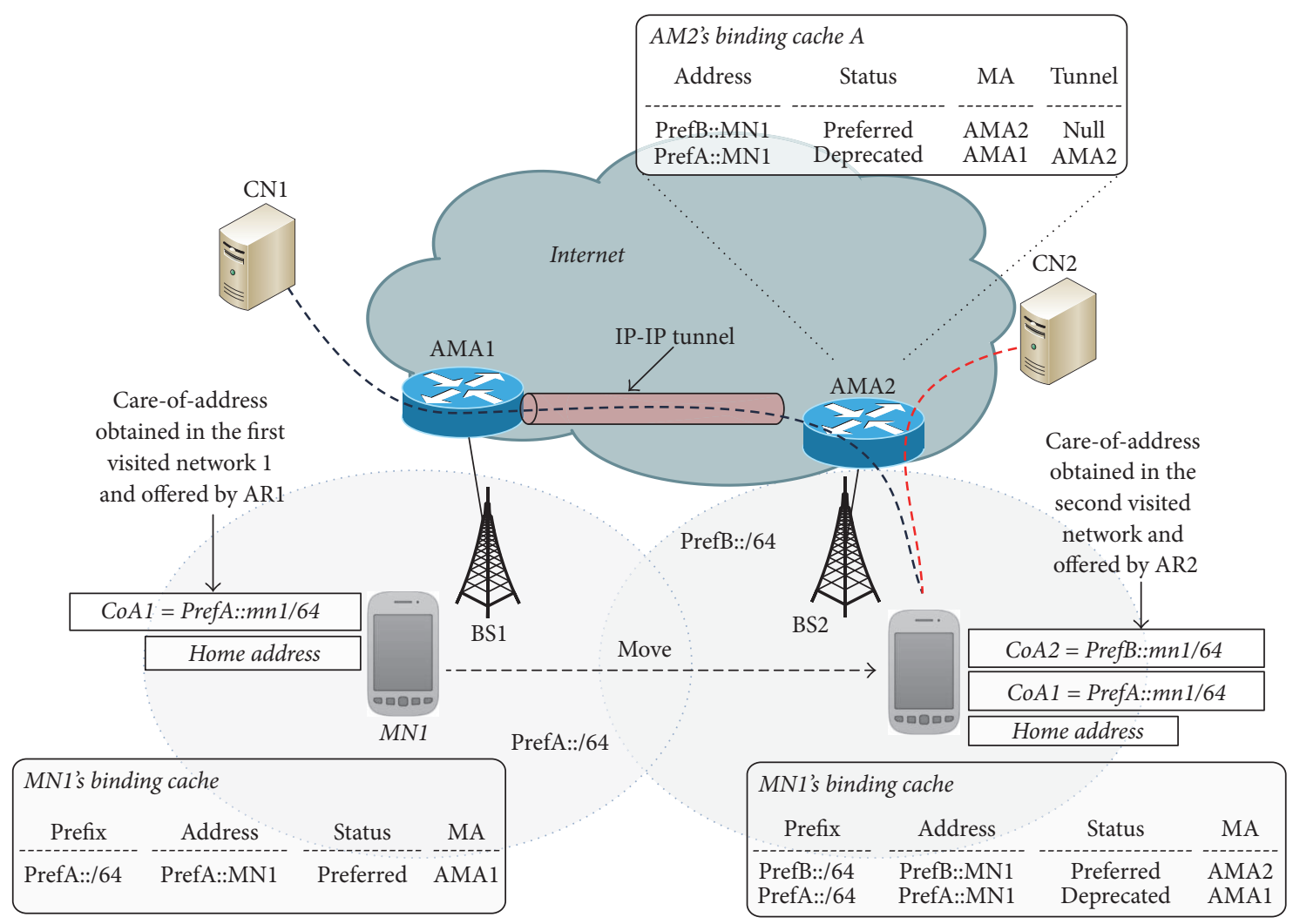

Figure 3: Host-Based DMM.

the edge of the access network level and the MN configures its address based on the provided network prefix from the AMA. When an MN moves to an adjacent access network, served by another AMA, a new address is configured in the MN based on the network prefix obtained at the new access network, while it keeps the previous address. As a result of the signaling between the serving AMA and the origin AMA, a bidirectional tunnel is created between them. As depicted in Figure 3, this solution creates multiple tunnels between AMAs and, in cases where a high mobility rate exists, the system performance might be critically compromised by the frequent registrations and maintenance of multiple tunnels.

Network-Based DMM (NB-DMM) [10] is a Distributed Mobility Management approach that has in common with PMIPv6 the fact that it is network-based; that is, it exempts the MN from participating in any mobility signaling, so no network software upgrade is required at the MN for mobility support because distributed mobility anchors perform mobility signaling on behalf of the MN. In NB-DMM, the mobility management functionalities are moved to the access routers level in order to anchor the traffic closer to the MN. Each $\mathrm{AR}$ is required to have both mobility anchoring and location functionalities, and it is referred to as a mobility capable access router (MAR). In NB-DMM, a new session is anchored at the current AR and initiated using the current IPv6 address. When a handover occurs before the end of the session, then the data traffic of this session is tunnelled between the current MAR and the anchoring MAR for this session. In order to achieve a network-based solution without the participation of the $\mathrm{MN}$ in the mobility signaling, the architecture is partially distributed and relies on a centralized database (Mobility Context DB). This DB stores ongoing sessions for the MNs. Thus, upon a handover, the new MAR retrieves the IP addresses of the anchoring MAR(s) for the MN's sessions from the database. Then, the new MAR proceeds to update the location by sending a PBU to each anchoring MAR. The basic operation of NB-DMM is depicted in Figure 4.

Additionally, we introduce DM3 [8]. This solution relies on a distributed architecture that is based on Mobile IPv6. The aim is to achieve efficient and flexible mobility management with QoS support taking advantage of both new Distributed Mobility Management approach and MPLS (Multiprotocol Label Switching) features. HB-DMM and NB-DMM proposals can suffer both tunnelling and signaling overhead in an environment where the mobile user moves frequently and creates new sessions during its movements. In that case, HB-DMM and NB-DMM establish an excessive number of tunnels that could make the solution inefficient. To provide more flexibility to the architecture, distributed anchor nodes in DM3, called Mobility Distributed Anchors (MDA), can be located in different places in the access network, in particular, not necessarily in the edge of the network. Thus, an MN will be anchored to a MDA when it ingresses the visited network. When a handover occurs, only the path from the MDA to the new AR will be modified and the data packets will be forwarded to the MN through the MDA. This way, the path is composed of a set of forwarding paths that will adapt to track host mobility and localize signaling in an area close to the 


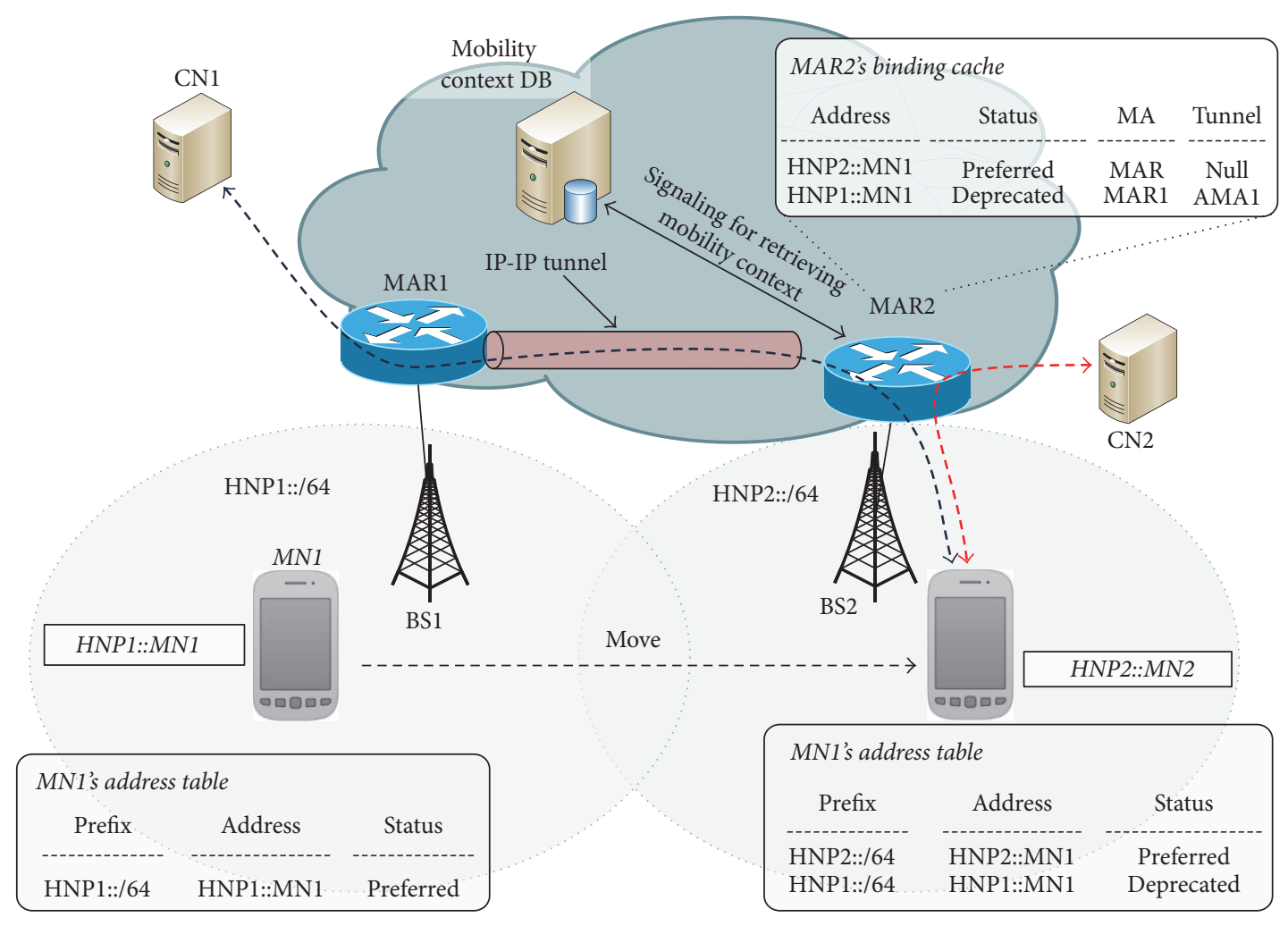

Figure 4: Network-Based DMM.

location of the MN. Figure 5 illustrates an overview of DM3 solution.

There have been some works evaluating the performance of DMM solutions and comparing them with centralized approaches. Particularly comprehensive are [12, 13]. Both highlight the advantages of DMM compared with centralized solutions. In our work, we also consider a DMM solution, DM3, which allows more flexible placement of anchor points, and accordingly analyze the effect of user movement on the performance of different mobility solutions.

Finally, other distributed solutions have been proposed for Network Mobility (NEMO) instead of host mobility. These NEMO proposals are out of the scope of this paper and are presented in detail in [14].

\section{Analytical Modelling}

Mobility, by its own nature, affects both control and data plane in the communication. When an MN moves, new signaling is introduced and a change in the routing path is needed in order to deliver the data packets to the new location of the MN. Moreover, mobility management protocols introduce tunnelling as an inherent mechanism to offer seamless mobility to the user. Under this framework, in this section, we evaluate the parameters involved in mobility aspects, such as the cost functions of traffic routing, registration updates, tunnelling overhead, handover latency, packet loss, and Mobility Anchor Load.
For the purpose of offering a complete analysis, we compare these metrics in both distributed (HB-DMM, NBDMM, and DM3) and centralized (MIPv6 and PMIPv6) mobility solutions. The parameters used in the analysis are shown in the Notations.

3.1. Signaling Cost. As we have described previously, one of the main functionalities for any IP mobility management protocol is the process of ensuring that the MN's mobility session is kept up to date while an MN moves among subnets. Such tasks require control messages that need to be sent among the mobility agents in the network. In general, a mobility management protocol requires that an $\mathrm{MN}$ sends a location update to its mobility anchor whenever it moves from one subnet to another. This location registration is required even though the $\mathrm{MN}$ does not communicate with others while moving. The signaling cost associated with location updates may become very significant as the number of MNs increases.

Moreover, this cost depends on the size of the signaling messages and the number of hops in every level 3 handover process during the time interval that the $\mathrm{MN}$ communication remains active. Thus, we refer to the aggregate signaling cost of registration update for a session as $C_{s}$. In MIPv6 and PMIPv6 cases, the registration update is needed with the mobility anchor (HA or LMA). Hence,

$$
\begin{gathered}
C_{s}^{\mathrm{MIP}}=2 \cdot s_{u} \cdot h_{\mathrm{MN}-\mathrm{HA}} \cdot N_{h} \\
C_{s}^{\mathrm{PMIP}}=2 \cdot s_{\mathrm{pu}} \cdot h_{\mathrm{MAG}-\mathrm{LMA}} \cdot N_{h} .
\end{gathered}
$$




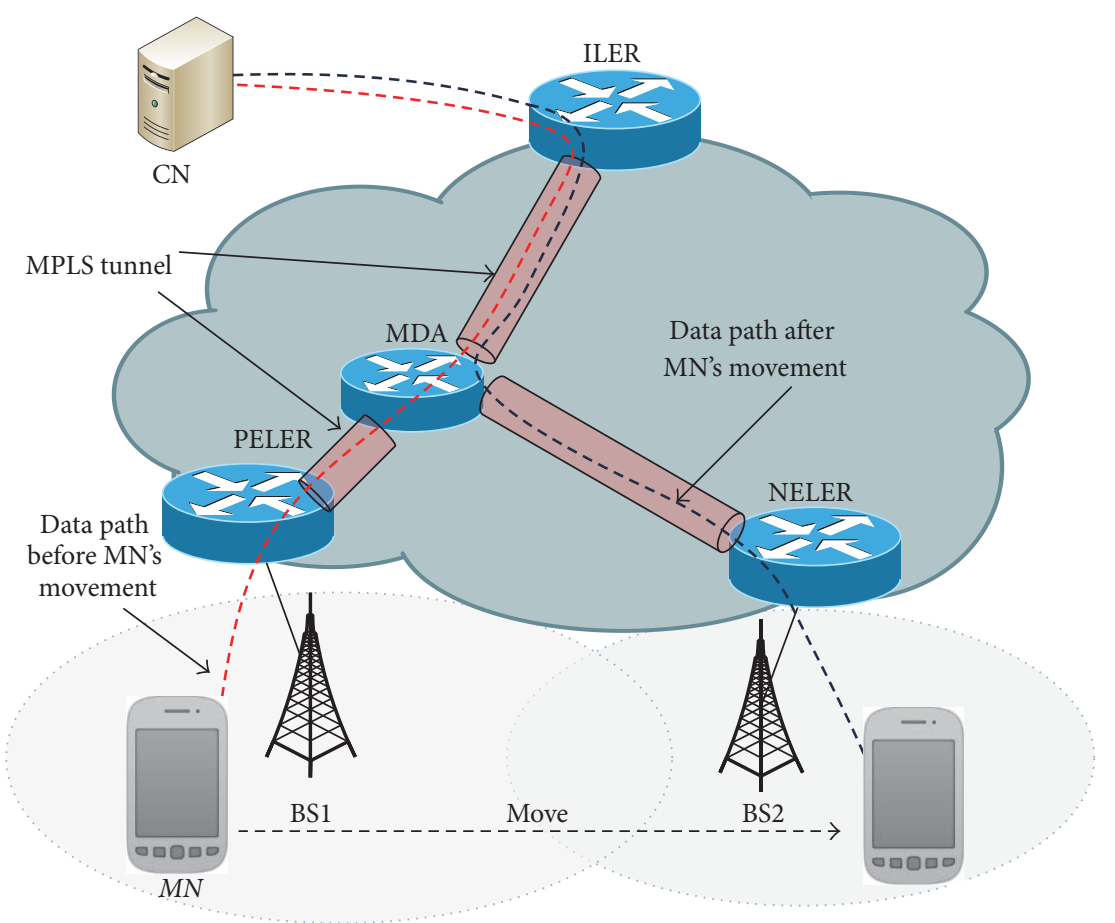

FIGURE 5: DM3 operation.

The distributed approaches, such as DM3, HB-DMM, and NB-DMM, update their movements with the distributed anchor, located closer to the location of the mobile node. In HB-DMM, the mobility anchor is called AMA and is located in the access router so AMA is the first IP capable router for the $\mathrm{MN}$. In this approach, when a mobile node moves, it registers its movement to the serving AMA (sAMA) that establishes bidirectional tunnels with previous AMA(s) which the MN was connected to. A similar phenomenon occurs with NB-DMM, where, upon handover, the new MAR retrieves the IP addresses of the anchoring MARs for the MN from the $\mathrm{DB}$. The new MAR then registers the $\mathrm{MN}$ at all these MARs. Apart from signaling the mobility management protocol, DM3 also adds the cost of LSP procedure setup. Let $n$ be the number of valid addresses configured at the $\mathrm{MN}$ in HB-DMM and NB-DMM ( $n=$ number of handover instances +1 ); assuming that the $\mathrm{MN}$ performs its handover while maintaining the communication sessions, we obtain the following values for the signaling cost in the distributed solutions:

$$
\begin{aligned}
C_{s}^{\mathrm{DM} 3}= & 2 \cdot s_{u} \cdot h_{\mathrm{MN}-\mathrm{MDA}} \cdot N_{h}+2 \cdot s_{l} \cdot h_{\mathrm{MDA}-\mathrm{NELER}} \cdot N_{h} \\
C_{s}^{\mathrm{HB}}= & {\left[2 \cdot s_{u} \cdot h_{\mathrm{MN}-\mathrm{sAMA}}+\sum_{i=1}^{n-1} 2 \cdot s_{u} \cdot h_{\mathrm{AMA}_{i} \text {-sAMA }}\right] } \\
& \cdot N_{h} \\
C_{s}^{\mathrm{NB}=} & {\left[2 \cdot s_{\mathrm{pu}} \cdot h_{\mathrm{AR}-\mathrm{DB}}+\sum_{i=1}^{n-1}\left(2 \cdot \mathrm{s}_{\mathrm{pu}} \cdot h_{\mathrm{MAR}_{i} \text {-sMAR }}\right)\right] } \\
& \cdot N_{h} .
\end{aligned}
$$

3.2. Data Packet Delivery Cost. The total data packet delivery cost for a session is defined as $C_{\mathrm{PD}}$. This value is influenced by the size of the data messages multiplied by the number of hops needed to forward packets from the $\mathrm{CN}$ to the $\mathrm{MN}$ and vice versa.

In MIPv6 and PMIPv6, packets are routed from the $\mathrm{CN}$ to the MN's anchor, HA or LMA, respectively, and then forwarded to the MN through a tunnel that encapsulates the data packets. Note that the packet delivery mode considered in MIPv6 is the bidirectional IP tunnelling, that is, without route optimization. Thus, the expressions that represent the cost can be expressed as follows:

$$
\begin{aligned}
& C_{\mathrm{PD}}^{\mathrm{MIP}}=\left(s_{d} \cdot h_{\mathrm{CN}-\mathrm{HA}}+\left(s_{t}+s_{d}\right) \cdot h_{\mathrm{HA}-\mathrm{MN}}\right) \cdot \lambda_{t} \cdot t_{s} \\
& C_{\mathrm{PD}}^{\mathrm{PMIP}}=\left(s_{d} \cdot h_{\mathrm{CN}-\mathrm{LMA}}+\left(s_{t}+s_{d}\right) \cdot h_{\mathrm{LMA}-\mathrm{MAG}}+s_{d}\right. \\
& \left.\cdot h_{\mathrm{MAG}-\mathrm{MN}}\right) \cdot \lambda_{t} \cdot t_{s} \cdot
\end{aligned}
$$

In HB-DMM and NB-DMM, when an MN moves, the traffic established in the new network will be routed directly to the $\mathrm{CN}$ whereas the remaining connections will be tunnelled to the MN's corresponding anchoring MAR and then routed to the $\mathrm{CN}$. $C_{\mathrm{PD}}$ in these distributed solutions is

$$
C_{\mathrm{PD}}^{\mathrm{HB}}=C_{\mathrm{PD}}^{\mathrm{NB}}=\left(P_{n} \cdot C_{\mathrm{PD}}^{d}+P_{h} \cdot C_{\mathrm{PD}}^{i}\right) \cdot \lambda_{t} \cdot t_{s},
$$

where $P_{n}$ and $P_{h}$ are, respectively, the probabilities that traffic is new or handover traffic. $P_{\mathrm{PD}}^{d}$ and $P_{\mathrm{PD}}^{i}$ are the units of cost 
of delivering one packet in the direct and indirect modes of DMM, respectively. Then, these costs are expressed as follows:

$$
\begin{aligned}
C_{\mathrm{PD}}^{d}= & s_{d} \cdot h_{\mathrm{CN}-\mathrm{sMAR}}+s_{d} \cdot h_{\mathrm{sMAR}-\mathrm{MN}} \\
C_{\mathrm{PD}}^{i}= & s_{d} \cdot h_{\mathrm{CN}-\mathrm{MAR}}+\left(s_{t}+s_{d}\right) \cdot h_{\mathrm{MAR}-\mathrm{sMAR}}+s_{d} \\
& \cdot h_{\mathrm{SMAR}-\mathrm{MN}} \cdot
\end{aligned}
$$

For its part, DM3 distributes mobility by means of MDA anchors in the access network, so data sent from the $\mathrm{CN}$ go to the MDA through the ILER and finally are delivered to the $\mathrm{MN}$. Hence, the value of packet delivery cost in DM3 is

$$
\begin{gathered}
C_{\mathrm{PD}}^{\mathrm{DM} 3}=\left(s_{d} \cdot h_{\mathrm{CN}-\mathrm{ILER}}+\left(s_{m}+s_{d}\right) \cdot h_{\mathrm{ILER}-\mathrm{MDA}}\right. \\
\left.+\left(s_{m}+s_{d}\right) \cdot h_{\mathrm{MDA}-\mathrm{MN}}\right) \cdot \lambda_{t} \cdot t_{s} .
\end{gathered}
$$

3.3. Tunnelling Cost. To achieve seamless mobility, all mobility protocols use a tunnel to forward packets. The tunnelling cost metric represents the cost of adding tunnelling overheads to the data packet delivery cost, so tunnelling cost $\left(C_{t}\right)$ can be derived from it by setting data packets size to zero, $s_{d}=$ 0 . In MIPv6 and PMIPv6, the traffic is tunnelled from the centralized anchor to the MN or the MAG agent. Hence,

$$
\begin{gathered}
C_{t}^{\mathrm{MIP}}=s_{t} \cdot h_{\mathrm{HA}-\mathrm{MN}} \cdot \lambda_{t} \cdot t_{s} \\
C_{t}^{\mathrm{PMIP}}=s_{t} \cdot h_{\mathrm{LMA}-\mathrm{MAG}} \cdot \lambda_{t} \cdot t_{s} .
\end{gathered}
$$

On the other hand, DMM solutions forward data traffic through a tunnel among the anchors. In DM3, MPLS technology is used instead of IPv6 over IPv6 tunnels. The reasoning behind the idea is that since a tunnel is needed, employing a technology that natively supports tunnelling seems to be a natural choice. Thus,

$$
\begin{aligned}
C_{t}^{\mathrm{HB}} & =s_{t} \cdot h_{\mathrm{AMA}-\mathrm{SAMA}} \cdot \lambda_{t} \cdot t_{s} \\
C_{t}^{\mathrm{NB}} & =s_{t} \cdot h_{\mathrm{MAR}-\mathrm{SMAR}} \cdot \lambda_{t} \cdot t_{s} \\
C_{t}^{\mathrm{DM} 3} & =\left(s_{l} \cdot h_{\mathrm{ILER}-\mathrm{MDA}}+s_{m} \cdot h_{\mathrm{MDA}-\mathrm{MN}}\right) \cdot \lambda_{t} \cdot t_{s} .
\end{aligned}
$$

3.4. Handover Latency and Packet Loss during a Session. Another relevant metric for mobility management protocols is the amount of packets lost during a session $\left(P_{\text {loss }}\right)$. This parameter is defined as the sum of lost packets per MN during all handover operations, and it depends on the handover latency. In this section, we analyze Packet Loss and Handover Latency for both the centralized and the distributed solutions. With respect to handover latency of mobility protocols, this metric is the sum of the layer 2 handover time $\left(T_{\mathrm{L} 2}\right)$, the movement detection time $\left(T_{\mathrm{MD}}\right)$, the authentication latency $\left(T_{\text {sec }}\right)$, and the time needed to exchange the mobility-related signaling $\left(T_{\text {reg }}\right)$. The operations leading to $T_{\mathrm{L} 2}$ are heavily dependent on the wireless technology deployed and do not actually depend on the layer 3 mobility protocol. $T_{\mathrm{MD}}$ is composed of exchanging the router solicitation (RS) and router advertisement (RA) messages between the MN and the new AR over the wireless link. The authentication tasks latency $\left(T_{\text {reg }}\right)$ depends on the security mechanisms which are not necessarily coupled with the mobility protocol. For fairness, we assume that both $T_{\mathrm{MD}}$ and $T_{\mathrm{sec}}$ are the same for all protocols. Thus, for handover latency, only the registration delay $\left(T_{\text {reg }}\right)$ depends on the mobility protocol and its specific procedures [10].

MIPv6 does not incorporate any buffering mechanism; thus, data packets sent from the $\mathrm{CN}$ to the $\mathrm{MN}$ will be lost while the MN performs its handover. Let $\lambda_{t}$ be the packet transmission rate; hence, this cost can be expressed as follows:

$$
P_{\text {loss }}^{\mathrm{MIP}}=T_{h}^{\mathrm{MIP}} \cdot \lambda_{t} \cdot N_{h}
$$

where $T_{h}^{\mathrm{MIP}}$ represents the handover delay due to the mobility management mechanisms of MIPv6. $T_{h}^{\mathrm{MIP}}$ can be written as

$$
T_{h}^{\mathrm{MIP}}=2 \cdot t\left(s_{u}, h_{\mathrm{MN}-\mathrm{HA}}\right) .
$$

Thus, packet loss can be defined as

$$
P_{\text {loss }}^{\mathrm{MIP}}=2 \cdot t\left(\mathrm{~s}_{u}, h_{\mathrm{MN}-\mathrm{HA}}\right) \cdot \lambda_{t} \cdot N_{h} \text {. }
$$

With respect to the packet loss in PMIPv6, the behavior is very similar to MIPv6, with the difference that the mobility bindings, necessary to configure the correct routing with the mobility anchor, are sent from the MAG to the LMA instead of from the MN. Hence, $P_{\text {loss }}^{\mathrm{PMIP}}$ can be expressed as follows:

$$
P_{\text {loss }}^{\text {PMIP }}=T_{h}^{\mathrm{PMIP}} \cdot \lambda_{t} \cdot N_{h} \text {, }
$$

where $T_{h}^{\mathrm{PMIP}}$ is

$$
T_{h}^{\mathrm{PMIP}}=2 \cdot t\left(s_{\mathrm{pu}}, h_{\mathrm{MAG}-\mathrm{LMA}}\right) .
$$

Thus, we have that packet loss in PMIPv6 is

$$
P_{\text {loss }}^{\text {PMIP }}=2 \cdot t\left(s_{\text {pu }}, h_{\mathrm{MAG}-\mathrm{LMA}}\right) \cdot \lambda_{t} \cdot N_{h} \text {. }
$$

Finally, the handover latency and packet loss in the distributed solutions (HB-DMM, NB-DMM, and DM3) are shown next. The distributed proposals require the serving mobility anchor (MAR and MDA) to exchange simultaneously the binding signaling with all previous anchors $\left(\mathrm{MAR}_{i}\right.$ and $\mathrm{MDA}_{i}$ ) that keep an active connection with the MN. Thus, these metrics are expressed as follows:

$$
\begin{aligned}
T_{h}^{\mathrm{HB}}= & 2 \cdot t\left(s_{u}, h_{\mathrm{MN}-\mathrm{AMA}}\right) \\
& +\max _{i=1}^{n-1}\left(2 \cdot t\left(s_{u}, h_{\mathrm{AMA}_{i} \text {-sAMA }}\right)\right) .
\end{aligned}
$$

Hence, packet loss in HB-DMM is

$$
P_{\text {loss }}^{\mathrm{HB}}=T_{h}^{\mathrm{HB}} \cdot \lambda_{t} \cdot N_{h} \text {. }
$$

Similar to the HB-DMM, the NB-DMM approach has an analogous operation during handover, with the additional requirement of retrieving the information from the database; thus,

$$
\begin{aligned}
T_{h}^{\mathrm{NB}}= & 2 \cdot t\left(s_{u}, h_{\mathrm{AR}-\mathrm{DB}}\right) \\
& +\max _{i=1}^{n-1}\left(2 \cdot t\left(s_{\mathrm{pu}}, h_{\mathrm{MAR}_{i} \text {-sMAR }}\right)\right) .
\end{aligned}
$$




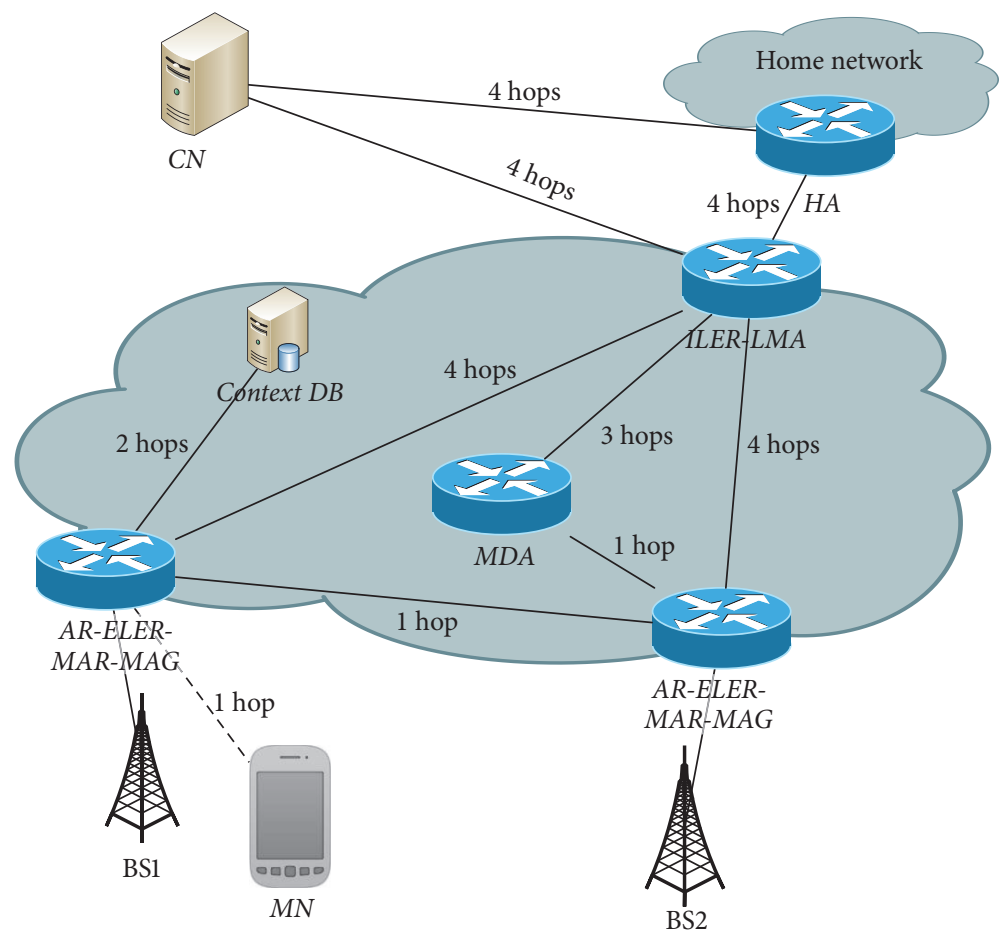

FIGURE 6: Distances in hops used in the analysis.

Thus, if a handover occurs, in-flight packets can be recovered and forwarded to the new location of the MN. The values of $T_{h}$ and $P_{\text {loss }}$ can be defined as

$$
\begin{aligned}
& T_{h}^{\mathrm{DM} 3}=2 \cdot t\left(s_{u}, h_{\mathrm{MN}-\mathrm{MDA}}\right)+2 \cdot t\left(s_{l}, h_{\mathrm{MDA}-\mathrm{NELER}}\right), \\
& P_{\mathrm{loss}}^{\mathrm{DM} 3}=t\left(s_{u}, h_{\mathrm{MN}-\mathrm{PELER}}\right) \cdot \lambda_{t} \cdot N_{h} .
\end{aligned}
$$

3.5. Mobility Anchor Load. In order to compare the load of a mobility anchor both in CMM and in DMM, a new metric is analyzed. Mobility Anchor Load $\left(C_{\mathrm{MAL}}\right)$ is defined as

$$
\begin{aligned}
C_{\mathrm{MAL}} & =\frac{\text { generated data by a MN }}{\text { number of mobility anchors }} \\
& =\frac{N_{s} \cdot \lambda_{s} \cdot t_{s} \cdot\left(N_{h}+1\right)}{N_{m}},
\end{aligned}
$$

where $N_{s}$ is the total number of communication sessions of an $\mathrm{MN}$ and $N_{m}$ is the number of mobility anchors associated with the MN. This parameter is $N_{m}=1$ in MIPv6 and PMIPv6 and $N_{m}=N_{h}+1$ in HB-DMM and NB-DMM. The value of this parameter in DM3 goes from $N_{m}=1 \cdots N_{h}$. Additional discussion about this parameter will be made in the next section.

\section{Numerical Investigations}

This section discusses the performance evaluation of MIPv6, PMIPv6, DM3, HB-DMM, and NB-DMM. Relative distances in hops for the numerical analysis are shown in Figure 6 and the default values of the system parameters are assumed to be as follows.

Traffic and network parameters are $s_{u}=56 \mathrm{~B}, s_{\mathrm{pu}}=76 \mathrm{~B}$, $s_{d}=1000 \mathrm{~B}, s_{t}=40 \mathrm{~B}, s_{l}=28 \mathrm{~B}, s_{m}=4 \mathrm{~B}, \lambda_{t}=8000 \mathrm{~B} / \mathrm{s}, t_{s}=$ $1000 \mathrm{~s}$, and $t_{r}=20 \mathrm{~s}$.

Figure 7 shows the comparison of signaling cost of registration update as a function of the cell residence time, which varies from 20 to 140 seconds. As could be expected, $C_{s}$ achieves the highest values when the cell residence time is low. In conditions of very high mobility (the cell residence time takes low values), only DM3 can significantly maintain an acceptable value for this parameter.

In fact, in DM3, this cost remains almost constant regardless of the mobility rate. Apart from DM3, in this zone of frequent handover, the behavior of DMM and CMM solutions can be easily distinguished. Both HB-DMM and NB-DMM reach the highest costs whereas the centralized solutions take intermediate values. This negative effect suffered by DMM solutions in scenarios of frequent movements among different subnets is due to the fact that the signaling messages in HB-DMM and NB-DMM solutions are exchanged among all the ARs that have been visited by the MN during its movement and an active connection remains. This complex address and tunnel management effect causes a significant increment in the signaling cost of the distributed protocols. However, the centralized solutions only need to notify the HA or LMA each time the MN moves to an adjacent network, minimizing the overall cost.

However, as cell residence time increments (the mobility of the nodes is lower), the behavior of signaling cost in 


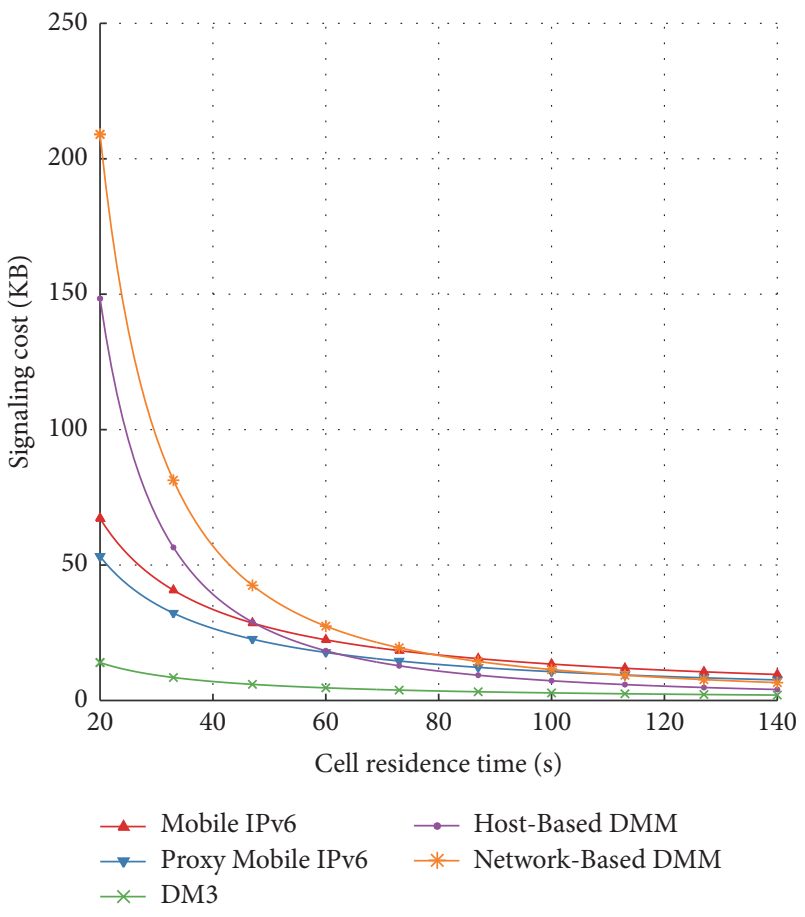

FIGURE 7: Signaling cost of registration updates.

DMM solutions decreases sharply and the cost remains in low values, even below the CMM protocols. This figure allows us to quantify the trade-off of the different proposals in the control plane.

Our DM3 solution uses the resources in the MPLS access network efficiently since it distributes the HA mobility functions in MDA nodes, not overloading links and nodes near the ILER. This way, DM3 can significantly reduce the registration cost particularly when the MN performs handoff frequently (i.e., the residence time in each subnet is short). The introduction of MDA nodes in the MPLS domain allows a reduction in the signaling exchange.

Data packet delivery cost represents the cost of delivering data packets to an MN per unit time. Figure 8 depicts the routing cost of forwarding data traffic during a session as a function of the transmission rate.

The results show that the packet delivery cost increases linearly with the transmission rate. As can be observed, distributed mechanisms outperform CMM solutions due to the operation of DMM protocols that avoid long routes and forward traffic in an optimized way. In centralized protocols, all packets are routed through a centralized anchor and this often results in longer paths from MN to $\mathrm{CN}$. The DM3 scheme obtains the best result, optimizing the data path by reducing an average of 35\% compared to MIPv6 and 21\% compared to PMIPv6. Furthermore, the DM3 solution has an average of $11 \%$ less packet delivery cost compared to the distributed solutions. This can be attributed to the fact that DM3 has a distributed control plane that allows each session to be anchored to a closer MDA.

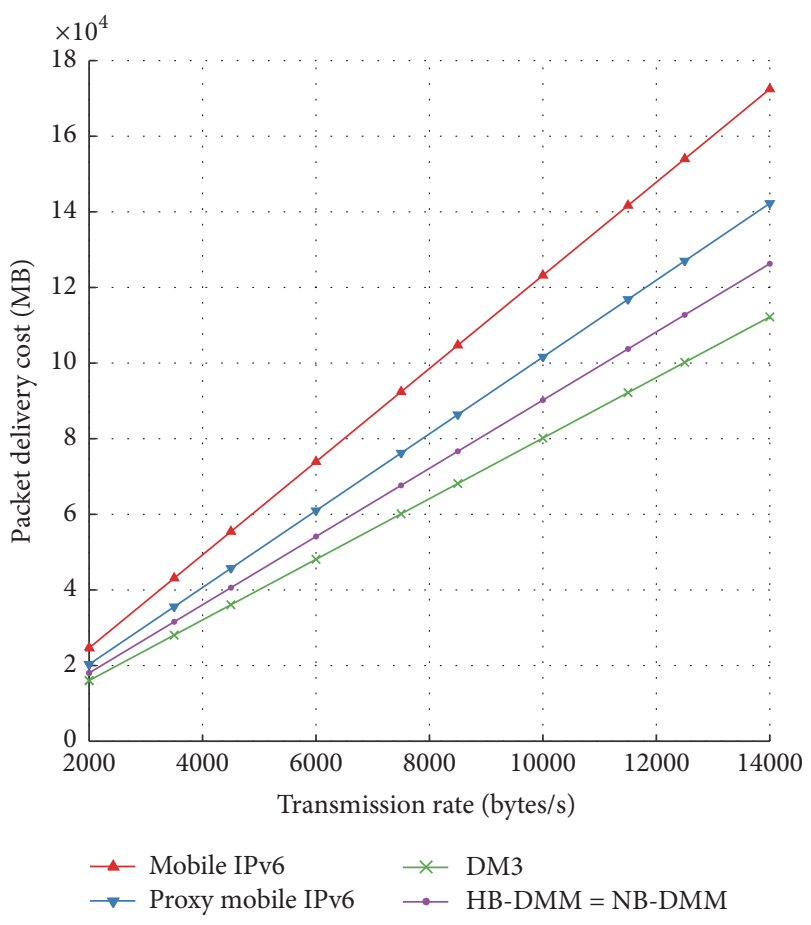

FIgURE 8: Packet delivery cost versus transmission rate.

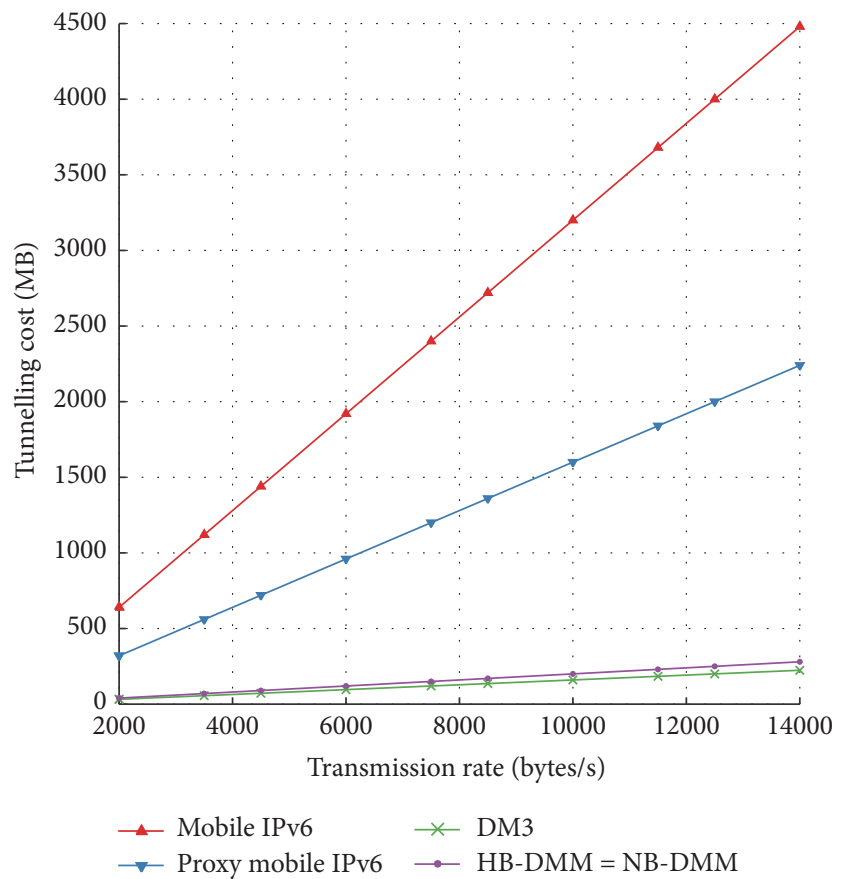

FIGURE 9: Tunnelling cost versus transmission rate.

Another metric closely related to packet delivery cost is the tunnelling cost. Figure 9 shows the variation of this cost as a function of the transmission rate.

It appears clearly that this cost in DM3 is lower than in the other proposals. This is due to the data path, which is more optimized than in CMM, and also due to the use of MPLS 


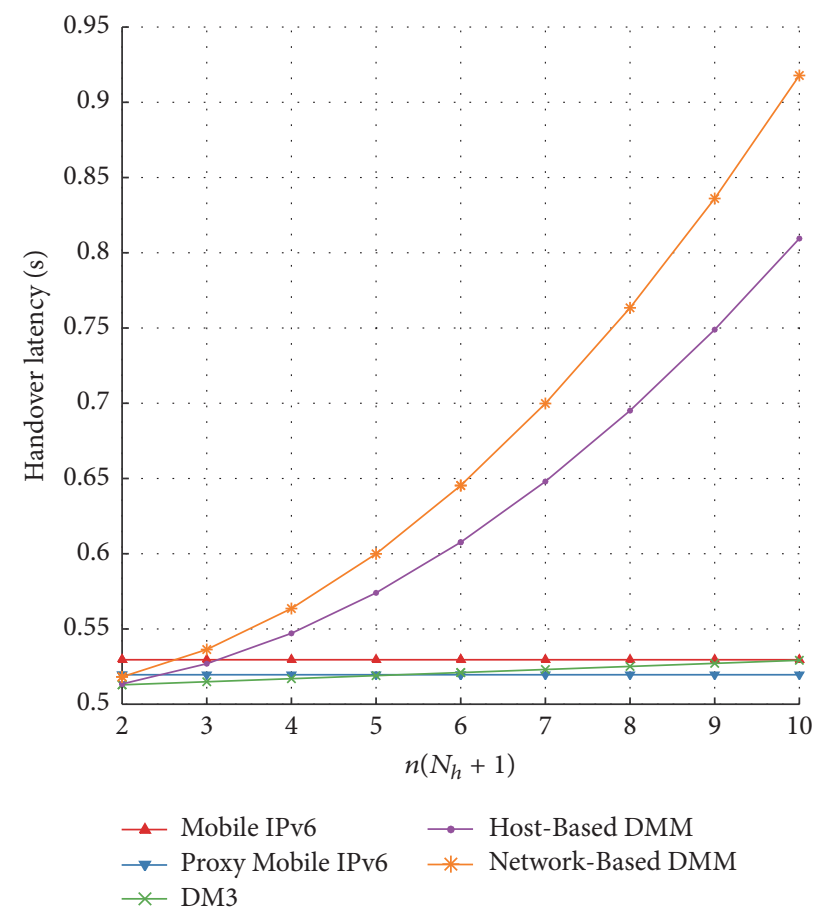

FIGURE 10: Handover latency.

tunnelling instead of IPv6 tunnelling. MPLS tunnels generate an overhead of 4 bytes, whereas IPv6 tunnels add an extra overhead of 40 bytes. For this reason, MIPv6 and PMIPv6 costs rise significantly with respect to DM3.

With respect to HB-DMM and NB-DMM, the MN initiates new sessions after the handover using the new IP address. The data traffic of these new sessions is routed in a more optimal way. As a result, the tunnelling cost is lower.

Next, the results of both handover latency and packet loss are shown. Figure 10 shows the variation of the handover latency as a function of $n=N_{h}+1$. In this case, the main difference in the handover latency of the mobility management protocols is the signaling exchanged during handover, necessary to maintain active ongoing IP flows when the MN changes its point of attachment to the network.

In CMM solutions, this metric mainly depends on the time needed for establishing new binding with the HA/LMA agent. Considering that all ARs are at the same distance from the centralized anchor, its handover latency is constant. The location management function in DM3 is located at the MDA nodes and a node is always anchored by the same MDA during all its session. That means that the AR needs to signal the MDA in order to complete a handover operation. That MDA is closer to the MN than the centralized anchor, so the handover latency of DM3 outperforms the centralized solutions when the MN performs few movements. Finally, the value of $n$ also has an impact in HB and NB-DMM proposals. Although the number of signaling messages required to perform handover in these mechanisms is high, the new MAR/AMA node performs the signaling with all the anchoring nodes simultaneously. Therefore, this value is given by the maximum time needed to perform the Binding Update with

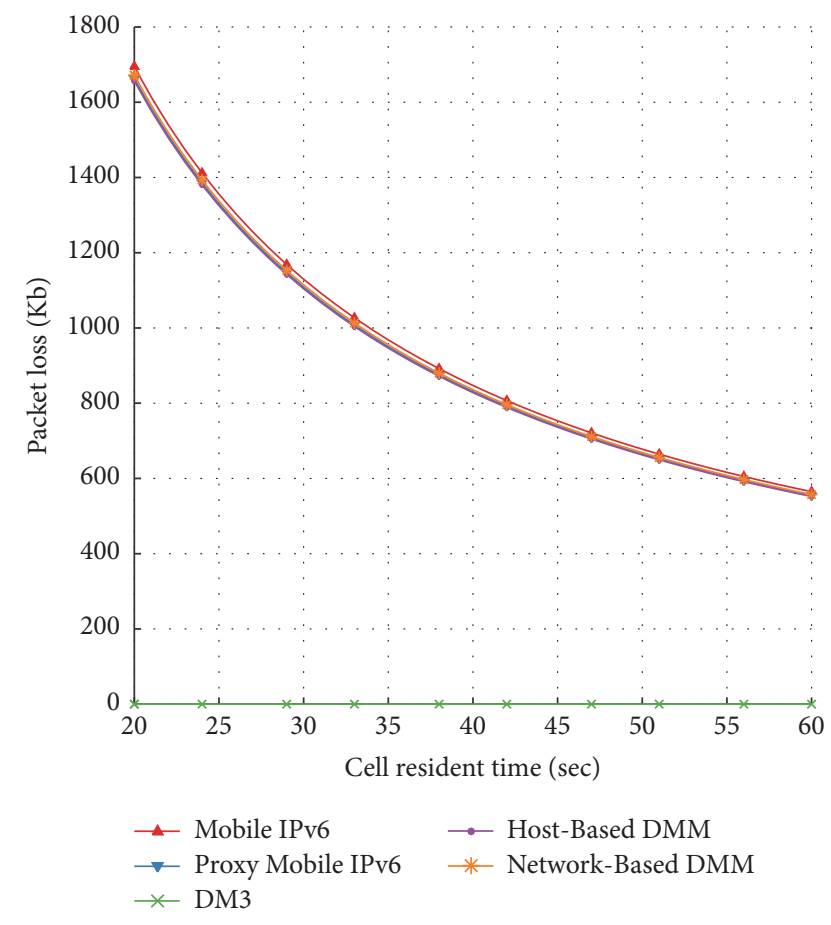

FIgURE 11: Packet loss during a session.

any anchoring node in which an active session remains. As can be seen in the figure, the distance between the $\mathrm{MN}$ and the distributed mobility anchor increases as the MN moves across the network. Thus, the dependency of DMM protocols is also shown on the number of movements made by the MN and, more specifically, on the number of connections that still remain established in previous cells.

Figure 11 shows the amount of packet loss during all of the connection sessions for each approach. These results show the large difference between the proposals which have buffering mechanisms and those which do not. Mobile IPv6, Mobile MPLS, and Host-Based DMM have the largest amount of packet loss due to the lack of a buffering mechanism during handover disruption time.

In order to minimize the packet loss, DM3 includes mechanisms to reduce losses. In all these solutions, the previous serving router of the $\mathrm{MN}$ is the one responsible for initiating the buffering mechanism. DM3 also achieves ordered delivery thanks to the recovery mechanism described in [8]. However, it is worth noting that handover latency in HB-DMM and NB-DMM is dependent on the number of connections as was depicted in Figure 10. Due to the impact of the handover latency in packet loss, the value of $N_{h}$ highly affects its behavior when the sessions remain active at previously visited networks. Thus, HB and NB-DMM solutions offer acceptable results when the relation between $N_{h}$ and session duration is high, but the packet losses rise dramatically when the number of tunnels established with current AMA/MAR increases.

Finally, the load of the mobility anchors is evaluated. Figure 12 shows the Mobility Anchor Load metric as the function of the number of sessions established by the MN $(n)$ at different access networks. 


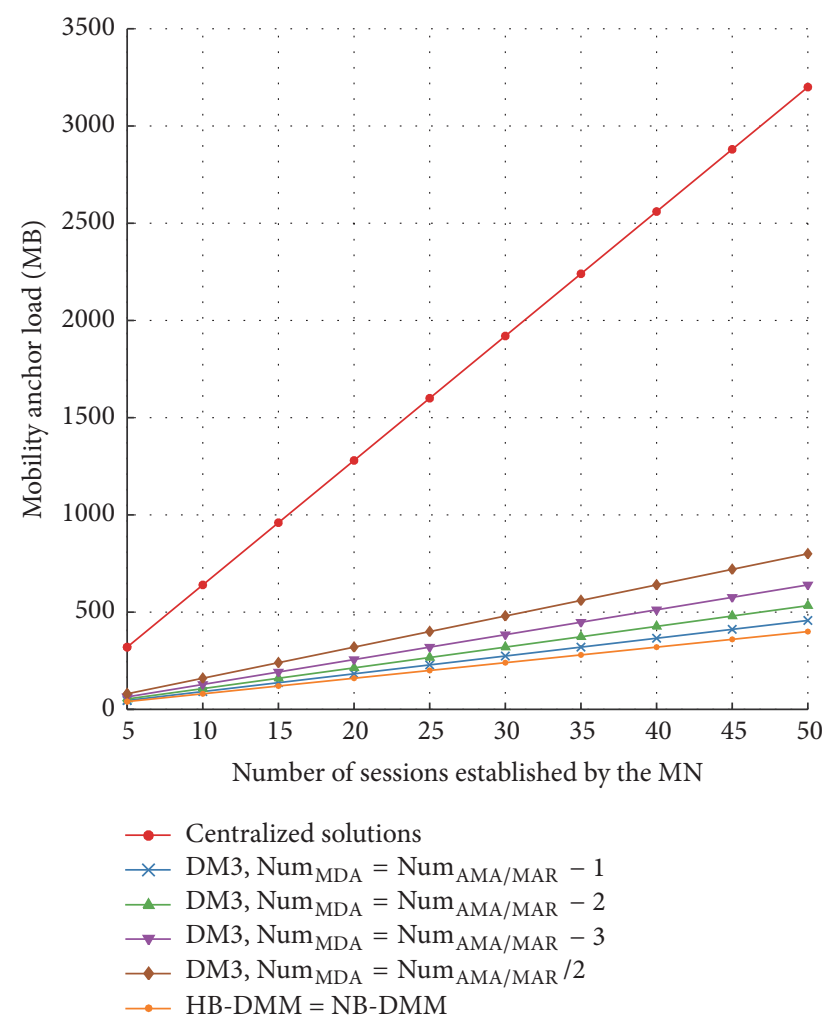

FIGURE 12: Mobility Anchor Load versus number of sessions by MN.

As $n$ increases, the load of mobility anchors of all protocols increases linearly, but at a different rate. In centralized mechanisms (MIPv6 and PMIPv6), the mobility anchor is responsible for all traffic forwarded from/to the MN so its load increases faster than in the distributed schemes. The load of HB-DMM, NB-DMM, and DM3 mobility anchors is lower because the traffic is processed in a distributed fashion and the failure impact of a mobility anchor among others is limited locally. Since the DM3 mobility anchors can be distributed at different levels of hierarchy, the DM3 behavior in these situations is outlined. This is distinct from HB-DMM and NB-DMM in which all mobility anchors (Num AMA/MAR $_{\text {AM }}$ ) are located in the access routers. Thus, DM3 can exhibit a different number of mobility anchors $\left(\mathrm{Num}_{\mathrm{MDA}}\right)$, depending on the hierarchy level in which they are located. Figure 12 also shows the mean traffic load of each mobility anchor in DM3 under these different configurations. It is observed that although HB-DMM and NB-DMM achieve the lowest load, DM3 also obtains moderate loads, even introducing only half the number of mobility anchors as in HB-DMM or NB-DMM $\left(\mathrm{Num}_{\mathrm{MDA}}=\mathrm{Num}_{\mathrm{AMA} / \mathrm{MAR}} / 2\right)$.

\section{Performance Evaluation through Simulation}

The evaluation through simulations aims to study IPv6 mobility management approaches in a more realistic environment than the one characterized by the analytical model, as well as validate the analytical results. The platform selected for the evaluation through simulations was MATLAB. An alternative would have been to use a network simulator, but MATLAB allows us to focus on the conceptual performance of the mobility solutions, independently of the implementation details of the network protocols. Next, the traffic model and the mobility patterns and the network topology used in the simulations are explained.

We consider a scenario where an MN may have simultaneous active sessions with several hosts $(\mathrm{CNs})$ in the Internet. We assume that sessions arrivals to an MN follow a Poisson process with mean rate $\lambda_{s}=0.01$ (i.e., the interarrival time between sessions is exponentially distributed with average (1/0.001) seconds). We assume also that the duration of a typical session is exponentially distributed with mean session duration $t_{s}=10$ time units.

Regarding the mobility of the MNs, we consider a Random Waypoint (RWP) mobility model, a simple and straightforward stochastic model. In RWP [15], a mobile node moves on a finite continuous plane from its current position to a new location by randomly choosing its destination coordinates, its speed of movement (from [minSpeed; maxSpeed]), and the amount of time that it will pause when it reaches the destination. On reaching the destination, the node pauses for some time which is distributed according to a random variable (from [minPause; maxPause]) and the process repeats itself. Once the pause time expires, the node chooses a new destination, speed, and pause time.

Apart from the Random Waypoint mobility, in order to drive the evaluation towards a more realistic scenario, we also run the simulation with real-world mobility track logs obtained from [16]. The sample settings where traces were obtained are two university campuses (one in Asia and one in the US), one metropolitan area (New York City), one state fair, and one theme park (Disney World). The participants walk most of the time and may also occasionally travel by bus, trolley, car, or subway. These settings are selected because they are conducive to collecting GPS readings.

Moreover, the simulation is run for a different number of MNs, ranging from 1 to 50 , and the simulation time is sufficiently large (45000 seconds) to avoid "typical runs" statistical problems. The dimension of the simulation scenario for the RWP mobility model is a rectangular area of $5 \times$ $5 \mathrm{~km}^{2}$ and the MNs are initially located randomly in the area. With regard to the real mobility tracks, the dimensions of the rectangular simulation area are set to be the same as in the GPS traces. In all simulation scenarios, we used the same initial positions found in the respective real traces for the same number of users. In the evaluation, the simulations are repeated 25 times to improve the accuracy of the results with a confidence interval of $95 \%$. Table 1 gives a summary of the setting values used in the simulations. The scenario defined for the evaluation is illustrated in Figure 13. In this topology, the leaf nodes act as access routers and the root node acts as the ingress node to the domain.

Due to dependence on the topology of DMM protocols, we selected this asymmetric topology due to its mixture 


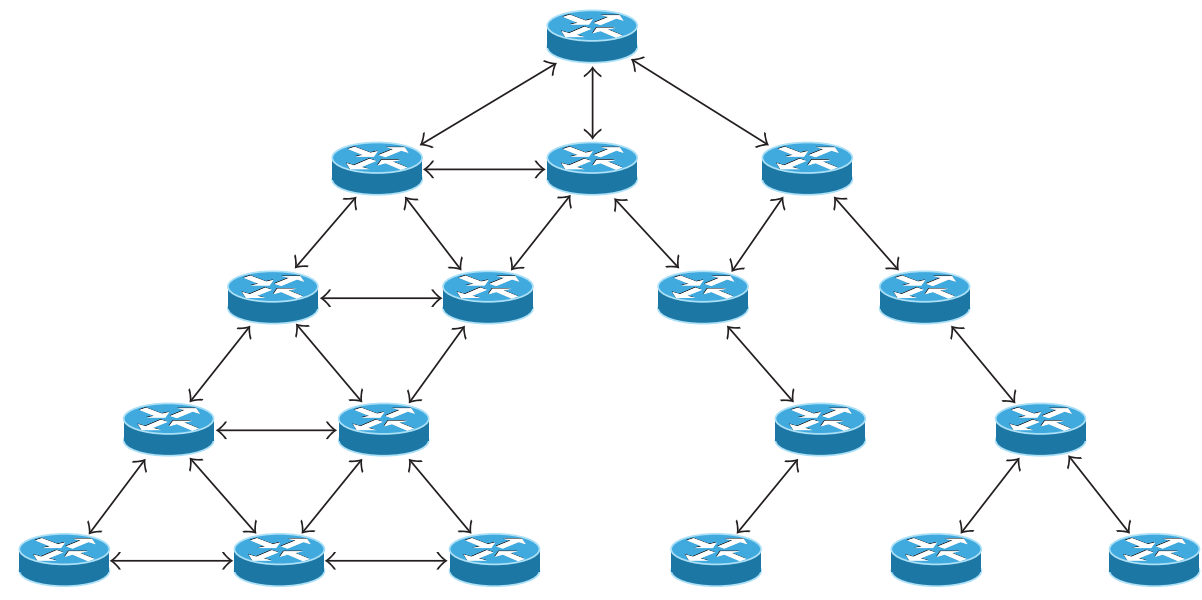

FIGURE 13: Topology used in the simulation.

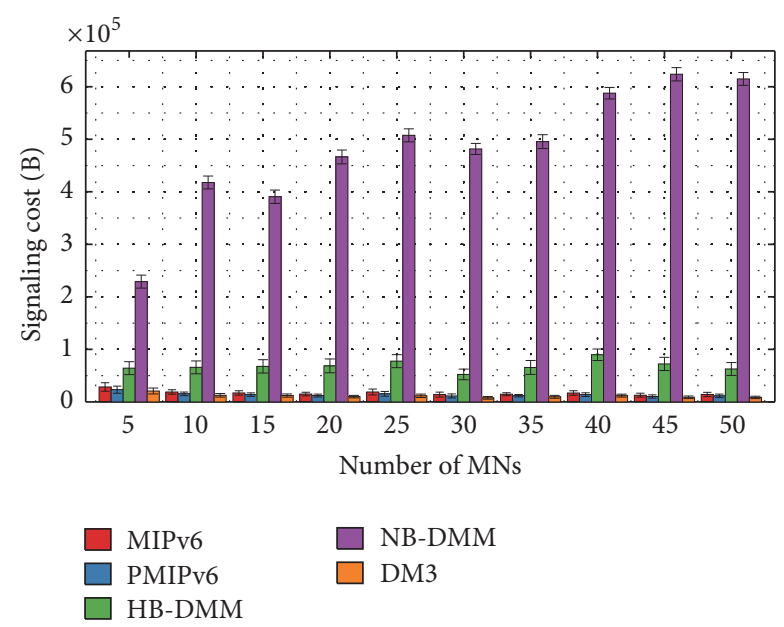

FIGURE 14: Signaling cost with a human-walk mobility model.

between a well-connected hierarchical network and a sparse network. This will produce more realistic results because the nodes will move around the connected and the sparse areas of the network, avoiding misleading performance by centralized or distributed protocols due to the network topology. In addition, such topology will allow us to shed further light on the dependency on network topology on the performance of different mobility management protocols.

Figures 14 and 15 show the comparison of accumulated signaling costs of registration update versus number of MNs during all the simulation executions.

In this case, HB-DMM and NB-DMM are penalized due to the high number of control messages that they need to send from the serving AR to all remaining mobility anchors in which the MN maintains an active session. Specifically, NB-DMM is the costlier protocol because of the necessity of sending an additional control message to the database each time a handover is made. This issue of high signaling cost in distributed solutions at high rates of mobility was also highlighted in Figure 7. Centralized solutions obtain

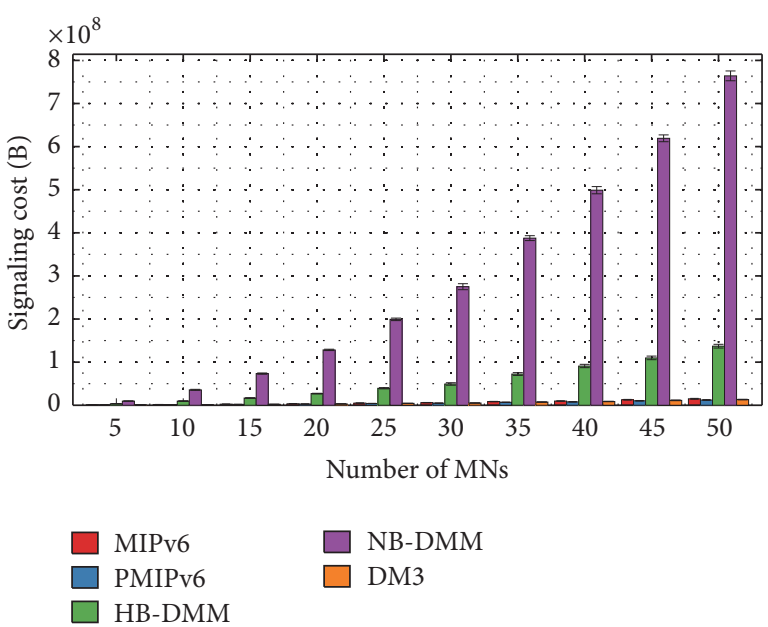

FIGURE 15: Signaling cost with a Random Waypoint mobility model.

TABLE 1: Simulation settings.

\begin{tabular}{lc}
\hline Parameter & Value \\
\hline Number of MNs & 5 to 50 \\
Number of ARs & 6 \\
Simulation time & $45000 \mathrm{~s}$ \\
Session arrival rate $\left(\lambda_{s}\right)$ & $0.01 \mathrm{~s}$ \\
Session duration $\left(t_{s}\right)$ & $10 \mathrm{~s}$ \\
MN movement model & RWP/human-walk traces \\
Simulation area & $5 \times 5 \mathrm{~km}^{2}$ \\
RWP speed interval & {$\left[\begin{array}{cc}1 & 10\end{array}\right] \mathrm{m} / \mathrm{s}$} \\
RWP pause interval & {$\left[\begin{array}{ll}60 & 300\end{array}\right] \mathrm{s}$} \\
RWP walk interval & {$\left[\begin{array}{cc}300 & 1200\end{array}\right] \mathrm{s}$} \\
Simulation run repetitions & 25 \\
\hline
\end{tabular}

lower signaling because in these protocols only two messages (Binding Update and Binding Acknowledgment) are needed to update all active sessions. DM3 is an intermediate solution since it distributes the HA mobility functions in MDA nodes, 


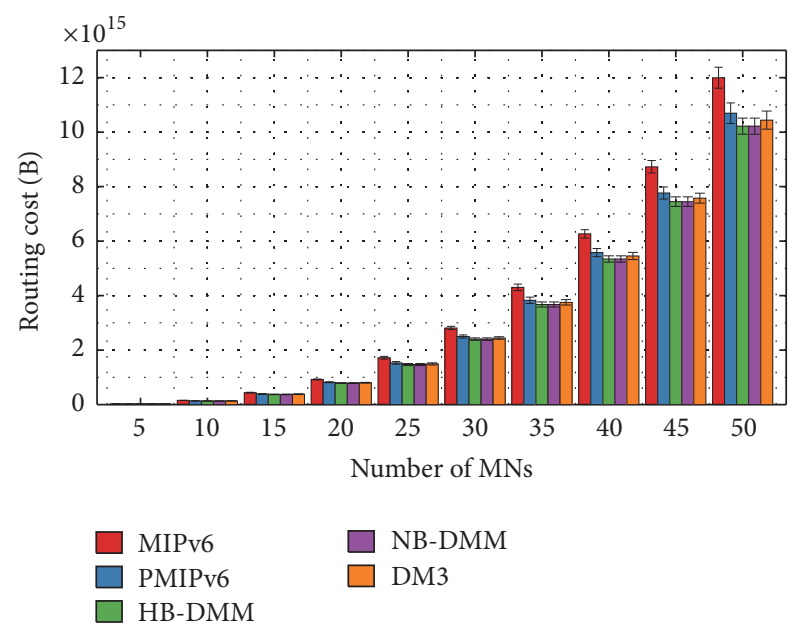

FIGURE 16: Packet delivery cost with a human-walk mobility model.

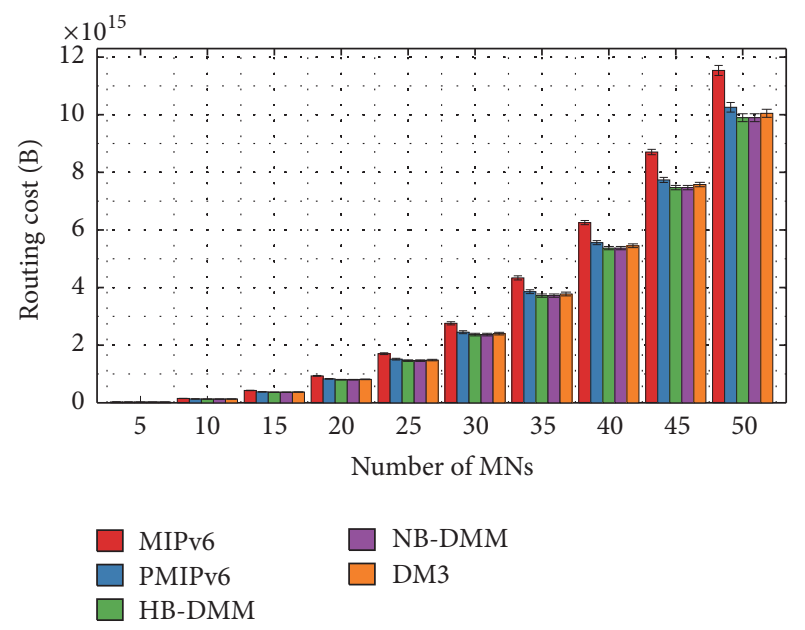

FIgURe 17: Packet delivery cost with a Random Waypoint mobility model.

not overloading links and nodes near the access routers. This way, DM3 can significantly reduce the registration cost of distributed mechanisms, achieving signaling overhead values similar to centralized protocols.

In Figures 16 and 17, we present the simulated results of accumulated packet delivery cost for different executions. In this case, HB-DMM and NB-DMM offer the same results because their data plane is similar, and the data are forwarded through the same path. In these figures, we can observe how centralized solutions perform nonoptimal routing and their cost is higher.

Finally, in Figures 18 and 19, the tunnelling costs of the mobility protocols are compared. The large difference between CMM and DMM solutions is highlighted. While HB-DMM and NB-DMM introduce an insignificant tunnelling cost, centralized solutions cause high overhead in the network due to the tunnelling process. Both HB-DMM and NB-DMM require tunnelling only between the distributed nodes, located at the access routers, whereas the tunnelling in centralized protocols is from the root of the domain. DM3 offers low values, close to the distributed solutions,

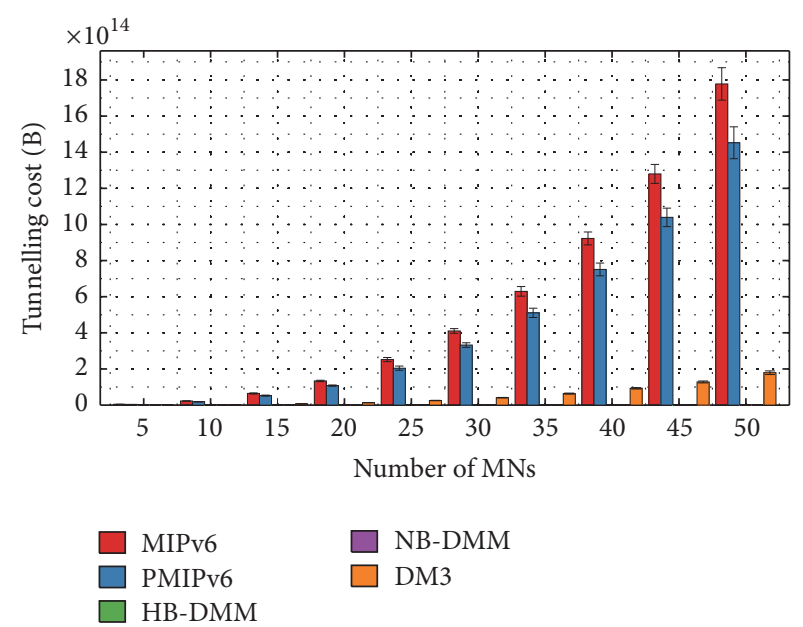

FIgURE 18: Tunnelling cost with a human-walk mobility model.

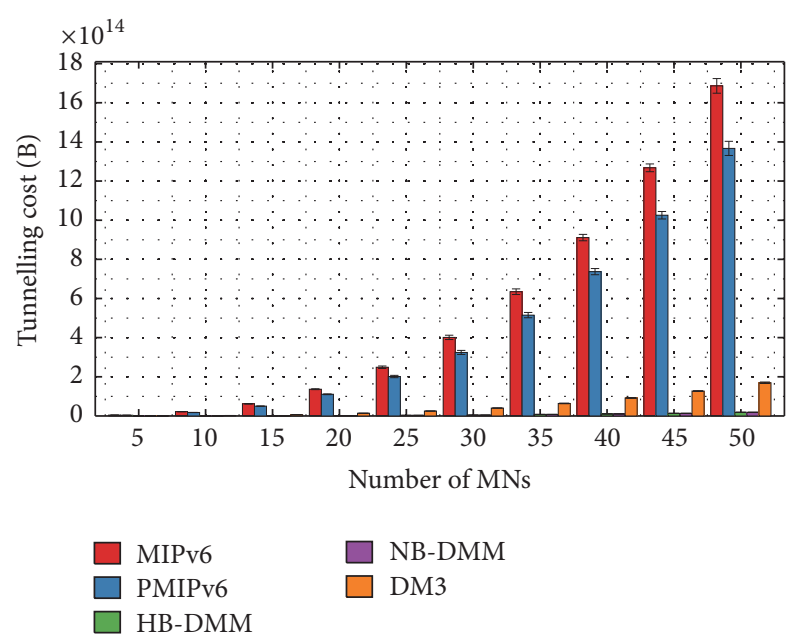

Figure 19: Tunnelling cost with a Random Waypoint mobility model.

and improves the tunnelling cost of centralized protocols significantly.

The simulation results shown in this section (signaling cost, packet delivery cost, and tunnelling cost) match the numerical analysis previously presented in Section 4 and are consistent with them, thus validating the analytical model. The DMM solutions face several limitations in terms of mobility signaling cost and this trend is also shown in the simulation results. With respect to the packet delivery cost, both analytical and simulation data follow the same pattern and DMM protocols reduce the cost with respect to centralized ones. This also occurs with the tunnelling cost, in which costs of distributed solutions are much less than the centralized ones in both cases.

\section{Conclusions}

Mobility management protocols are evolving towards a distributed operation in order to deal with increasing mobile 
Internet traffic demand. DMM solutions rely on distributed anchors, where mobility functions are located. With this scheme, the traffic utilizes significantly less overhead in terms of encapsulation, while scalability and robustness issues are improved. Although DMM generally helps to save the resources in the network, there are a number of scenarios in which the operation of DMM might lead to a reduction in performance.

In this context, this article is focused on an exhaustive analytic and simulated evaluation of representative CMM and DMM approaches which allows further light to be shed on these approaches and compares the DMM approaches with existing CMM protocols in terms of both control and data plane metrics. We have derived the analytic expressions of the most relevant mobility costs such as signaling cost, packet delivery cost, tunnelling cost, handover latency, packet loss, and Mobility Anchor Load.

Our results reveal the limitations of DMM solutions in some specific scenarios, especially those in which cell residence time is short and/or the number of remaining active sessions in previous networks is high. Thus, based on the evaluations undertaken in this paper, we can conclude that when an $\mathrm{MN}$ moves frequently and it begins new sessions in different visited networks, the performances of HB-DMM and NB-DMM approaches fall down due to the number of tunnels that need to be managed by the distributed nodes which also depends on network topology. In other cases, DMM is more efficient than CMM. To solve these DMM drawbacks, DM3 locates the mobility anchor in the access network and reduces the number of tunnels that need to be established. DM3 can be seen as a balanced solution that improves the performance of the data and signaling plane.

In the next step, we consider that future mobile network architectures might benefit from using hybrid CMM-DMM solutions, in which operators would be able to handle the traffic in a distributed or centralized way depending on different relevant factors such as application requirements in terms of mobility management, traffic patterns, or network load.

\section{Notations}

$\begin{array}{ll}s_{u}: & \text { Size of the BU message } \\ s_{\mathrm{pu}}: & \text { Size of the PBU message } \\ s_{l}: & \text { Size of a message for LSP establishment } \\ s_{d}: & \text { Average size of a data packet } \\ s_{t}: & \text { Average size of the IPv6 tunnel header } \\ s_{m}: & \text { Average size of the MPLS tunnel header } \\ \lambda_{t}: & \text { Transmission rate } \\ \lambda_{s}: & \text { Interarrival time between sessions } \\ t_{s}: & \text { Total session time } \\ N_{h}: & \text { Number of L3 handover operations in a } \\ h_{x-y}: & \text { session } \\ t\left(s, h_{x-y}\right): & \text { Timerage hop distance between } x \text { and } y \\ & \text { the path between } x \text { and } y .\end{array}$

\section{Competing Interests}

The authors declare that there are no competing interests regarding the publication of this paper.

\section{Acknowledgments}

This work was supported by the Government of Extremadura under Grant no. GR15099 and by the European Regional Development Fund Programme (2014-2020) and the Regional Fund, through Computing and Advanced Technologies Foundation of Extremadura (COMPUTAEX).

\section{References}

[1] Cisco Systems Inc, Cisco Visual Networking Index: Global Mobile Data Traffic Forecast Update, 2015-2020, Cisco, 2016.

[2] Nokia Siemens Networks, "Signaling is growing $50 \%$ faster than data traffic," White Paper, 2012.

[3] C. Perkins, D. Johnson, and J. Arkko, "Mobility Support in IPv6," RFC Editor RFC6275, 2011.

[4] S. Gundavelli, K. Leung, V. Devarapalli, K. Chowdhury, and B. Patil, "Proxy mobile IPv6," RFC 5213, IETF, 2008.

[5] H. Chan, D. Liu, P. Seite, H. Yokota, and J. Korhonen, "Requirements for Distributed Mobility Management," IETF RFC 7333, August 2014.

[6] J. C. Zuniga, C. J. Bernardos, A. De La Oliva, T. Melia, R. Costa, and A. Reznik, "Distributed mobility management: a standards landscape," IEEE Communications Magazine, vol. 51, no. 3, pp. 80-87, 2013.

[7] D. Liu, J. C. Zuniga, P. Seite, H. Chan, and C. J. Bernardos, "Distributed Mobility Management: Current Practices and Gap Analysis," IETF RFC 7429, January 201.

[8] J. Carmona-Murillo, J.-L. González-Sánchez, D. Cortés-Polo, and F.-J. Rodríguez-Pérez, "DM3: distributed mobility management in MPLS-based access networks," International Journal of Network Management, vol. 24, no. 2, pp. 85-100, 2014.

[9] J.-H. Lee, J.-M. Bonnin, P. Seite, and H. A. Chan, "Distributed IP mobility management from the perspective of the IETF: motivations, requirements, approaches, comparison, and challenges," IEEE Wireless Communications, vol. 20, no. 5, pp. 159168, 2013.

[10] H. Ali-Ahmad, M. Ouzzif, P. Bertin, and X. Lagrange, "Performance analysis on network-based distributed mobility management," Wireless Personal Communications, vol. 74, no. 4, pp. 1245-1263, 2014.

[11] K.-S. Kong, W. Lee, Y.-H. Han, M.-K. Shin, and H. You, "Mobility management for all-IP mobile networks: mobile IPv6 vs. proxy mobile IPv6," IEEE Wireless Communications, vol. 15, no. 2, pp. 36-45, 2008.

[12] S. Jeon, N. Kang, D. Corujo, and R. L. Aguiar, "Comprehensive performance evaluation of distributed and dynamic mobility routing strategy," Computer Networks, vol. 79, pp. 53-67, 2015.

[13] F. Giust, C. J. Bernardos, and A. De La Oliva, "Analytic evaluation and experimental validation of a network-based IPv6 distributed mobility management solution," IEEE Transactions on Mobile Computing, vol. 13, no. 11, pp. 2484-2497, 2014.

[14] P. P. Ernest, O. E. Falowo, and H. A. Chan, "Design and performance evaluation of distributed mobility management schemes for network mobility," Journal of Network and Computer Applications, vol. 61, pp. 46-58, 2016. 
[15] E. Hyytiä, P. Lassila, and J. Virtamo, "Spatial node distribution of the random waypoint mobility model with applications," IEEE Transactions on Mobile Computing, vol. 5, no. 6, pp. 680694, 2006.

[16] I. Rhee, M. Shin, S. Hong, K. Lee, and S. Chong, "On the levy-walk nature of human mobility," in Proceedings of the 27th IEEE Communications Society Conference on Computer Communications (INFOCOM '08), pp. 1597-1605, Phoenix, Ariz, USA, April 2008. 

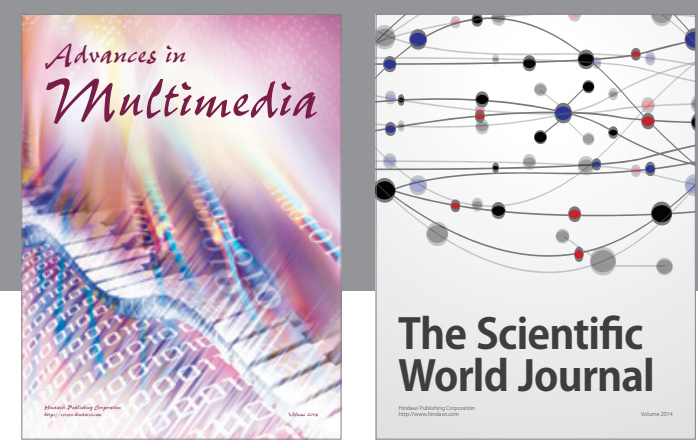

The Scientific World Journal
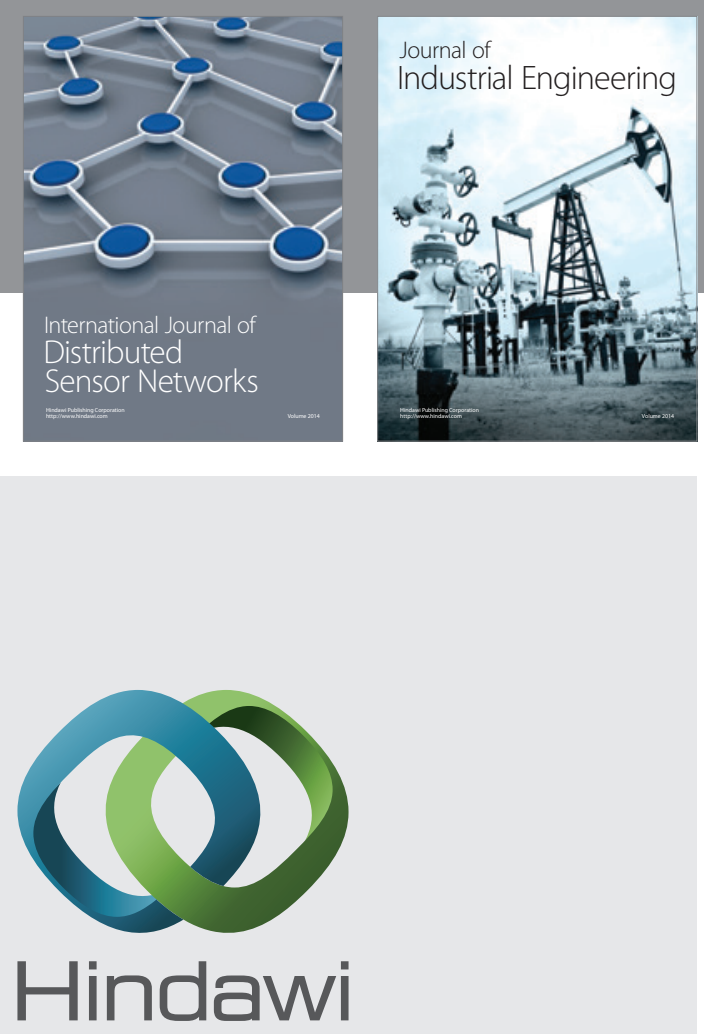

Submit your manuscripts at

https://www.hindawi.com

\section{Computer Networks} and Communications
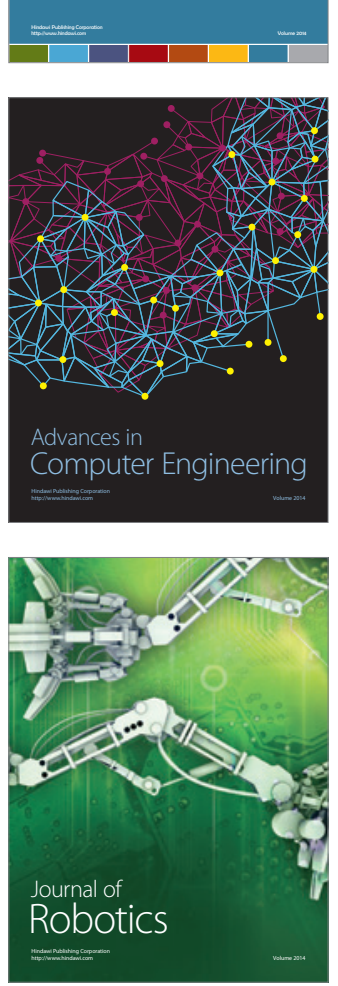
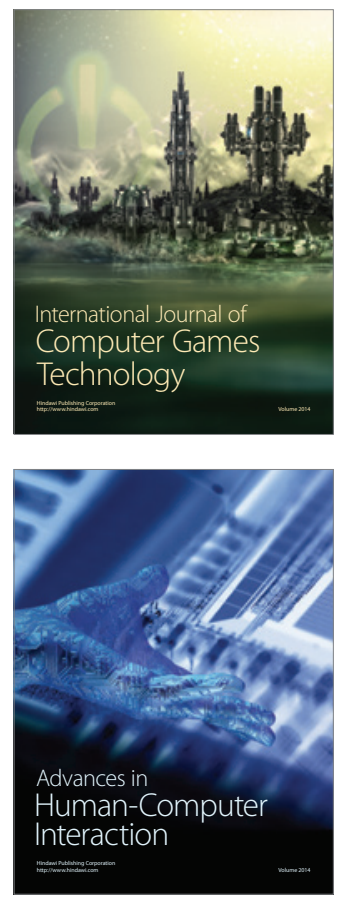
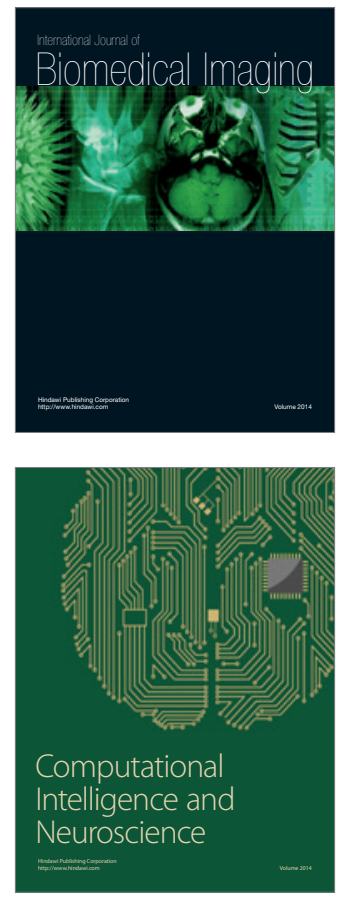
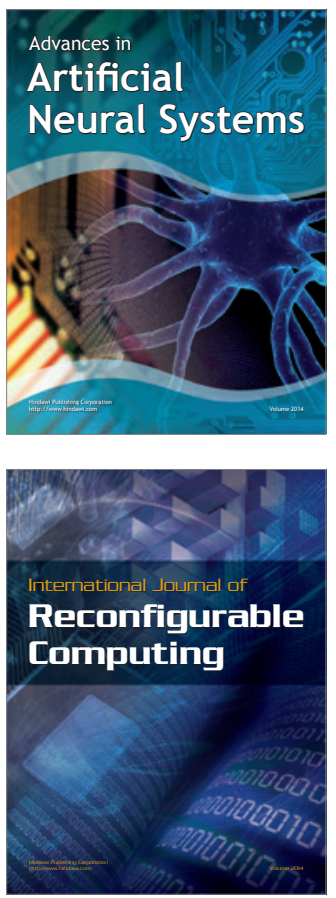
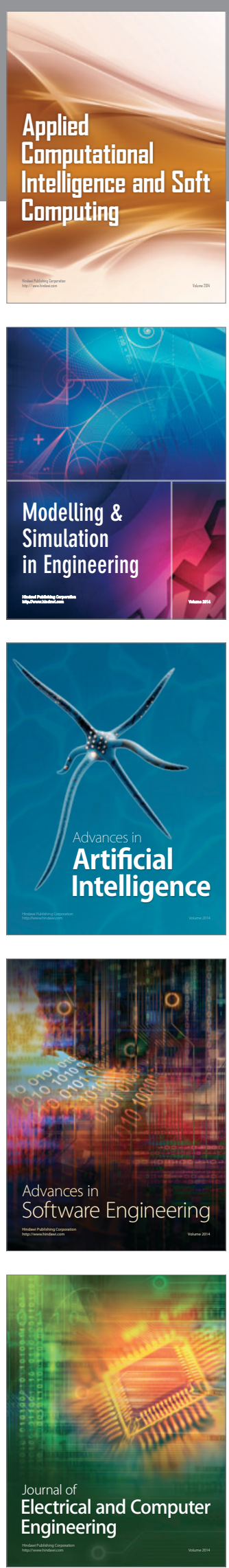